\title{
Resummed thermodynamic perturbation theory for bond cooperativity in associating fluids
}

\author{
Bennett D. Marshall ${ }^{\mathrm{a})}$ and Walter G. Chapman \\ Department of Chemical and Biomolecular Engineering, Rice University, 6100 S. Main, Houston, \\ Texas 77005, USA
}

(Received 18 September 2013; accepted 11 November 2013; published online 3 December 2013)

\begin{abstract}
We develop a resummed thermodynamic perturbation theory for bond cooperativity in associating fluids by extension of Wertheim's multi-density formalism. We specifically consider the case of an associating hard sphere with two association sites and both pairwise and triplet contributions to the energy, such that the first bond in an associated cluster receives an energy $-\varepsilon^{(1)}$ and each subsequent bond in the cluster receives an energy $-\varepsilon^{(2)}$. To test the theory we perform new Monte Carlo simulations for potentials of this type. Theory and simulation are found to be in excellent agreement. We show that decreasing the energetic benefit of hydrogen bonding can actually result in a decrease in internal energy in the fluid. We also predict that when $\varepsilon^{(1)}=0$ and $\varepsilon^{(2)}$ is nonzero there is a transition temperature where the system transitions from a fluid of monomers to a mixture of monomers and very long chains. ( 2013 AIP Publishing LLC. [http://dx.doi.org/10.1063/1.4834637]
\end{abstract}

\section{INTRODUCTION}

The hydrogen bond is a fundamental interaction whose influence is felt throughout all areas of science. Hydrogen bonding is responsible for the remarkable properties of water, ${ }^{1}$ folding of proteins ${ }^{2}$ and has been extensively employed in the supramolecular assembly of synthetic polymers. $^{3}$ The distinguishing features of the hydrogen bond (we will interchangeably use association) are that it is short ranged and anisotropic resulting in bond saturation. Wertheim $^{3-7}$ incorporated these general features of the hydrogen bond into a rigorous statistical mechanical formalism by introduction of a multi-density approach where each bonding state of a molecule was treated as a distinct species and assigned a density. This approach allowed Wertheim to regroup the resulting cluster expansion in such a way that bond saturation was naturally and exactly included in the theory. Wertheim's multi-density approach is typically applied as a perturbation theory ${ }^{8}$ with a hard sphere reference fluid. In first order (TPT1) each association site is treated independently resulting in a simple equation of state ${ }^{9}$ which has been extensively employed as a model for hydrogen bonding fluids in both academia and industry. ${ }^{10}$ In addition to homogeneous fluids, Wertheim's theory has also found widespread application to interfacial fluids in the form of classical density functional theory. ${ }^{11}$

A major assumption, which lays the foundation of Wertheim's approach, is that hydrogen bonding is pairwise additive, there is no cooperativity. However, as has become increasingly apparent in recent years, hydrogen bond cooperativity plays a significant role in many physical processes. Both hydrogen fluoride $(\mathrm{HF})^{12}$ and alcohols ${ }^{13}$ have been shown to

\footnotetext{
a) Author to whom correspondence should be addressed. Electronic mail: bennettd1980@gmail.com
}

exhibit strong hydrogen bond cooperativity. In addition, hydrogen bond cooperativity has been shown to stabilize peptide hydrogen bonds ${ }^{14}$ and has recently been implicated ${ }^{15}$ in the anomalous low temperature phase behavior of water.

To account for bond cooperativity in an equation of state a number of lattice theories ${ }^{16,17}$ have been developed. In an alternative approach, Sear and Jackson (SJ) ${ }^{18}$ considered a potential model of a 2 site associating fluid where the first bond in an associating cluster was given an association en$\operatorname{ergy}-\varepsilon^{(1)}$ and each remaining bond was given an association energy $-\varepsilon^{(2)}$. This model approximates chains of hydrogen bonds in HF, where it has been shown that the binding energy per hydrogen bond decreases as cluster size increases. This continues until a chain length of approximately six, at which point the bond energy stabilizes as chain length increases. ${ }^{12}$ Using this model $\mathrm{SJ}^{18}$ developed a theory in the associating ideal gas limit and then used this ideal form of the theory to construct, in an ad hoc fashion, the equation of state at higher densities. This theory has never been validated against molecular simulations and the effect of varying the magnitudes and ratios of the energies $\varepsilon^{(1)}$ and $\varepsilon^{(2)}$ was never extensively explored. For instance, do spheres associate when $\varepsilon^{(1)}$ is small and $\varepsilon^{(2)}$ is large?

With the wide ranging success of Wertheim's multidensity approach in modeling bulk and interfacial hydrogen bonding fluids which exhibit pairwise additivity, it would be prudent to incorporate hydrogen bond cooperativity into the formalism. The purpose of this paper is threefold. First we will extend Wertheim's multi-density formalism to account for bond cooperativity. As a first step we will consider the same two site case considered by SJ. ${ }^{18}$ We derive the theory in an intuitive manner using a resummed perturbation theory where we consider bond cooperativity as a perturbation. Second, we will test this new theory and the theory of SJ against Monte Carlo simulation results, and third we will discuss extensions of the theory to other, more complex, systems. 


\section{THEORY}

In this section we develop the resummed thermodynamic perturbation theory for bond cooperativity in two site associating fluids with a single type $A$ and type $B$ association site. We restrict association such that there are $A B$ attractions but no $A A$ or $B B$ attractions. We follow $\mathrm{SJ}$ and consider a fluid composed of $N_{P}$ hard spheres of diameter $d$ with two association sites $A$ and $B$ with a total energy composed of pairwise and triplet contributions, ${ }^{18}$

$$
\begin{aligned}
U\left(1 \ldots N_{P}\right)= & \frac{1}{2} \sum_{i, j}\left(\phi_{H S}\left(r_{i j}\right)+\phi_{a s}^{(2)}(i j)\right) \\
& +\frac{1}{6} \sum_{i, j, k} \phi_{a s}^{(3)}(i j k),
\end{aligned}
$$

where (1) $=\left\{\vec{r}_{1}, \Omega_{1}\right\}$ represents the position $\vec{r}_{1}$ and orientation $\Omega_{1}$ of sphere 1 and $\phi_{H S}$ is the hard sphere reference potential. The terms $\phi_{a s}^{(2)}(i j)$ and $\phi_{a s}^{(3)}(i j k)$ are the pairwise and triplet association contributions and are given by ${ }^{18}$

$$
\begin{aligned}
\phi_{a s}^{(2)}(i j)= & -\varepsilon^{(1)}\left(\mathrm{O}_{A B}(i j)+\mathrm{O}_{B A}(i j)\right), \\
\phi_{a s}^{(3)}(i j k)= & -\left(\varepsilon^{(2)}-\varepsilon^{(1)}\right)\left(\mathrm{O}_{A B}(i j) \mathrm{O}_{B A}(i k)\right. \\
& +\mathrm{O}_{B A}(i j) \mathrm{O}_{A B}(i k) \\
& +\mathrm{O}_{A B}(j i) \mathrm{O}_{B A}(j k)+\mathrm{O}_{B A}(j i) \mathrm{O}_{A B}(j k) \\
& \left.+\mathrm{O}_{A B}(k i) \mathrm{O}_{B A}(k j)+\mathrm{O}_{B A}(k i) \mathrm{O}_{A B}(k j)\right),
\end{aligned}
$$

where $\mathrm{O}_{A B}(i j)$ is the overlap function which, in this paper, we obtain using conical square well association sites ${ }^{19-21}$

$$
\mathrm{O}_{A B}(i j)=\left\{\begin{array}{cc}
1 & r_{12} \leq r_{c} \text { and } \theta_{\mathrm{A}} \leq \theta_{c} \text { and } \theta_{\mathrm{B}} \leq \theta_{c} \\
0 & \text { otherwise }
\end{array},\right.
$$

which states that if spheres $i$ and $j$ are within a distance $r_{c}$ of each other and each sphere is oriented such that the angles between the site orientation vectors and the vector connecting the two spheres, $\theta_{\mathrm{A}}$ for sphere $i$ and $\theta_{\mathrm{B}}$ for sphere $j$, are both less than the critical angle $\theta_{c}$ the two sites are considered bonded. See Fig. 1 for an illustration. The triplet contribution $\phi_{a s}^{(3)}$ serves to add a correction $-\left(\varepsilon^{(2)}-\varepsilon^{(1)}\right)$ for each sphere bonded twice.

Before considering the case with bond cooperativity, we will review Wertheim's $N$ th order perturbation theory $(\mathrm{TPTN})^{8}$ for pairwise additive association. For this two site case the Helmholtz free energy in Wertheim's theory with the

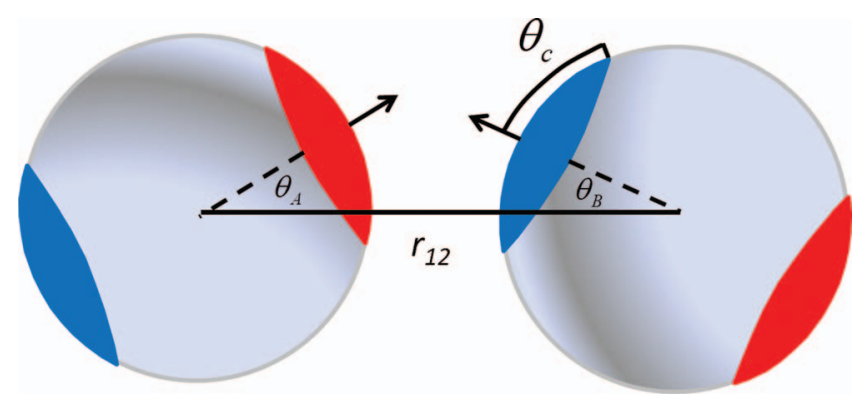

FIG. 1. Diagram of pair interactions between two associating spheres. pairwise additivity assumption is given as ${ }^{8}$

$$
\frac{A-A_{H S}}{k_{B} T V}=\rho \ln \frac{\rho_{o}}{\rho}-\sigma_{A}-\sigma_{B}+\frac{\sigma_{A} \sigma_{B}}{\rho_{o}}+\rho-\frac{\Delta c^{(o)}}{V} .
$$

Here $T$ is the temperature, $k_{B}$ is the Boltzmann constant, $\rho$ is the total density, $\rho_{o}$ is the monomer density, and $\sigma_{A}=\rho_{A}$ $+\rho_{o}$ where $\rho_{A}$ is the density of molecules bonded at only site $A$. There is a similar relation for $\sigma_{B}$. The term $V$ is the system volume and $A_{H S}$ is the free energy of the hard sphere reference system. Finally, $\Delta c^{(o)}$ is the associative contribution to the fundamental graph sum. In this paper we will consider associating spheres as shown in Fig. 1, where the association sites are located on opposite poles of the sphere. Since the separation between sites is large, we can neglect the possibility of ring formation and double bonding. ${ }^{22}$ Also, we will consider potential parameters $\theta_{c}$ and $r_{c}$ such that multiple bonding of an association site cannot occur due to steric hindrance. In TPT $N, \Delta c^{(o)}$ is approximated by considering all chain diagrams which contain a single chain of $N$ or less association bonds and is given as ${ }^{8}$

$$
\Delta c^{(o)}=\sum_{n=1}^{N} \Delta c_{n}
$$

where $\Delta c_{n}$ is the $n$th order contribution (involves chains of $n$ association bonds) and is given by

$$
\frac{\Delta c_{n}}{V}=\sigma_{A} \sigma_{B} \rho_{o}^{n-1} I_{n}
$$

The integrals $I_{n}$ are given by

$$
\begin{aligned}
I_{n}= & \frac{1}{\Omega^{n}} \int f_{A B}(12) \ldots f_{A B}(n, n+1) G(1 \ldots n+1) d(2) \\
& \ldots d(n+1),
\end{aligned}
$$

where $\Omega=4 \pi$ (for our axisymmetric case) and the $f_{A B}(i j$ ) are the association Mayer functions. Wertheim defines the functions $G(1 \ldots n+1)$ as, "the subset of graphs in $g(1 \ldots n$ +1 ) such that combining them with the chain produces an irreducible graph; $g(1 \ldots s)$ denotes the $s$ particle correlation function of the reference system." 8 This means, for instance, that in a second order perturbation theory the contribution $\Delta c_{2}$ will include the triplet correlation function $g(123)$, but one must subtract off the contribution from the first order term $\Delta c_{1}$ to keep from double counting. We then obtain the $G(1 \ldots s)$ by summing $g(1 \ldots s)$ and all products of $g$ 's obtained by partitioning $1 \ldots \mathrm{s}$ into subsequences which share the switching point and associating $\mathrm{a}-1$ with each switching point. ${ }^{8}$ A few examples include

$$
\begin{aligned}
G(12)= & g(12), \\
G(123)= & g(123)-g(12) g(23), \\
G(1234)= & g(1234)-g(123) g(34)-g(12) g(234) \\
& +g(12) g(23) g(34) .
\end{aligned}
$$

The general idea of TPTN is then to build up chains by adding in higher order contributions and subtracting off lower order contributions. What does this have to do with bond cooperativity?

Now we wish to approximate the associative graph sum for fluids which exhibit bond cooperativity as given in 


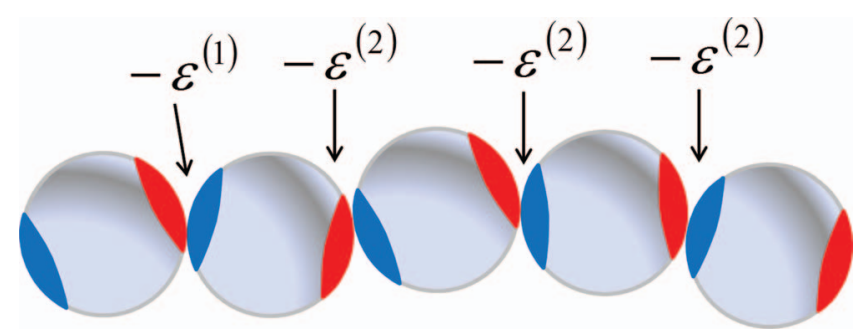

FIG. 2. Diagram of bond energy distributions in an associated cluster.

Eqs. (1) and (2). For this case the first bond in a cluster receives an energy $-\varepsilon^{(1)}$, while each remaining bond receives an energy $-\varepsilon^{(2)}$. This is illustrated in Fig. 2. Wertheim's multi-density formalism, and cluster expansions in general, rely on the assumption of pairwise additivity. To include triplet correlations in the form of bond cooperativity we will construct $\Delta c^{(o)}$ in a single chain approximation using generalized Mayer functions. Instead of using TPTN to enforce intra-cluster steric constraints, we will employ an analogous perturbation theory to incorporate bond cooperativity. For the case of a chain which exhibits bond cooperativity as given in Eqs. (1) and (2), one should expect the product of Mayer functions in a chain of length $s$ to be

$$
\tilde{f}(1 \cdots s)=f_{A B}^{(1)}(12) f_{A B}^{(2)}(23) \cdots f_{A B}^{(2)}(s-1, s),
$$

where we have introduced the Mayer functions $f_{A B}^{(j)}(12)$ defined by

$$
f_{A B}^{(j)}(12)=\left(\exp \left(\varepsilon^{(j)} / k_{B} T\right)-1\right) \mathrm{O}_{A B}(12)=f_{A B}^{(j)} \mathrm{O}_{A B}(12) .
$$

Since we are considering the two site case with axial symmetry, steric hindrance between sites is small and the correlation functions $g(1 \cdots s)$ can be approximated by a linear superposition (no bond angle dependence as in Ref. 22)

$$
g(1 \cdots s)=\prod_{k=1}^{s-1} g(k, k+1) .
$$

Combining Eqs. (9) and (11) one might conclude that the chain integral $I_{n}$ for this case (similar to Eq. (7) but for bond cooperativity) would be given by

$$
\frac{1}{\Omega^{n}} \int \tilde{f}(1 \cdots n+1) g(12) \cdots g(n, n+1) d(2) \ldots d(n+1) .
$$

However, Eq. (12) is incorrect due to the fact we did not properly subtract off the lower order contributions to keep from overcounting, as was discussed above in the context of TPTN. To account for this we must subtract off all the ways a chain of $n$ spheres could be created from chains containing $m<n$ spheres. Proper accounting results in the following chain integral:

$$
\begin{aligned}
I_{n}= & \frac{1}{\Omega^{n}} \int \tilde{F}(1 \cdots n+1) g(12) \cdots g(n, n+1) d(2) \\
& \ldots d(n+1),
\end{aligned}
$$

where $\tilde{F}(1 \cdots s)$ is defined by the same partition properties of $G(1 \cdots s)$ with the sole difference being the exchange $g(1 \cdots s) \rightarrow \tilde{f}(1 \cdots s)$. For instance $\tilde{F}(12)$

$$
\begin{aligned}
=\tilde{f}(12)= & f_{A B}^{(1)} \mathrm{O}_{A B}(12) \text { and } \\
\tilde{F}(123)= & \tilde{f}(123)-\tilde{f}(12) \tilde{f}(23) \\
= & f_{A B}^{(1)}\left(f_{A B}^{(2)}-f_{A B}^{(1)}\right) \mathrm{O}_{A B}(12) \mathrm{O}_{A B}(23), \\
\tilde{F}(1234)= & \tilde{f}(1234)-\tilde{f}(123) \tilde{f}(34)-\tilde{f}(12) \tilde{f}(234) \\
& +\tilde{f}(12) \tilde{f}(23) \tilde{f}(34) \\
= & f_{A B}^{(1)}\left(f_{A B}^{(2)}-f_{A B}^{(1)}\right)^{2} \mathrm{O}_{A B}(12) \mathrm{O}_{A B}(23) \mathrm{O}_{A B}(34) .
\end{aligned}
$$

For the general case $\tilde{F}(1 \cdots s)$ we obtain the following relation:

$$
\tilde{F}(1 \cdots s)=f_{A B}^{(1)}\left(f_{A B}^{(2)}-f_{A B}^{(1)}\right)^{s-2} \prod_{k=1}^{s-1} \mathrm{O}_{A B}(k, k+1) .
$$

In constructing the chain integrals for the case of bond cooperativity we have essentially made a change of variables as compared to the standard TPTN. In its standard form, TPTN corrects for multi-body $(>2)$ effects in relation to intracluster repulsions by introduction of higher order correlation functions. In the development of Eq. (13), we have assumed multi-body effects associated with intra-cluster repulsions are small, and used an analogous TPTN to correct for multibody effects in the association energies in the form of the association Mayer functions. We have simply switched the roles of intra-cluster attractions and repulsions in reference to higher order corrections to the perturbation theory. The standard TPTN is built on a methodical theoretical footing, where our approach here is intuitive. Further justification of our approach will be given by excellent agreement with simulation predictions.

The form of Eq. (15) allows for a complete summation over all chain graphs. Finally, we write the new fundamental graph sum for an $N$ th order perturbation theory for bond cooperativity and let the order of perturbation become infinitely large $N \rightarrow \infty$ to obtain

$$
\frac{\Delta c^{(o)}}{V}=\sigma_{A} \sigma_{B} \sum_{n=1}^{\infty} \rho_{o}^{n-1} I_{n}=\frac{\sigma_{A} \sigma_{B} f_{A B}^{(1)} \Delta}{1-\left(f_{A B}^{(2)}-f_{A B}^{(1)}\right) \rho_{o} \Delta},
$$

where $\Delta=\pi\left(1-\cos \theta_{c}\right)^{2} \xi$ and $\xi=\int_{d}^{r_{c}} r^{2} g(r) d r$ which we approximate $\operatorname{as}^{23} \xi \approx d^{2}\left(r_{c}-d\right) g(d)$. The term $g(d)$ is the hard sphere reference system pair correlation function at contact which is obtained using the Carnahan and Starling fitting function. ${ }^{24}$ Equation (16) is the central result of this paper and is remarkably simple. We have developed a new perturbation theory where we use perturbations to correct for bond cooperativity. We then allowed the order of perturbation to become infinitely large allowing for a summation over all chain contributions. For the case $\varepsilon^{(2)}=\varepsilon^{(1)}$ the standard first order perturbation theory ${ }^{8}$ is recovered. Using Eq. (16) we can minimize the free energy Eq. (4) with respect to $\sigma_{B}$ and $\rho_{o}$ to obtain the mass action equations

$$
\frac{\sigma_{A}}{\rho_{o}}-1=\frac{\sigma_{A} f_{A B}^{(1)} \Delta}{1-\left(f_{A B}^{(2)}-f_{A B}^{(1)}\right) \rho_{o} \Delta}
$$


and

$$
\begin{aligned}
\frac{\rho}{\rho_{o}}= & \left(\frac{\sigma_{A}}{\rho_{o}}\right)^{2}+\left(f_{A B}^{(2)}-f_{A B}^{(1)}\right) f_{A B}^{(1)} \\
& \times\left(\frac{\sigma_{A} \Delta}{1-\left(f_{A B}^{(2)}-f_{A B}^{(1)}\right) \rho_{o} \Delta}\right)^{2},
\end{aligned}
$$

where $\sigma_{A}=\sigma_{B}$ due to symmetry. Using Eq. (17) we can simplify the free energy as

$$
\frac{A-A_{H S}}{k_{B} T V}=\rho \ln \frac{\rho_{o}}{\rho}-\sigma_{A}+\rho .
$$

Combining (17) and (18) we obtain a closed equation for $\rho_{o}$

$$
\frac{\rho}{\rho_{o}}=1+\frac{2 \rho_{o} f_{A B}^{(1)} \Delta}{1-\rho_{o} f_{A B}^{(2)} \Delta}+\frac{\left(\rho_{o} \Delta\right)^{2} f_{A B}^{(1)} f_{A B}^{(2)}}{\left(1-\rho_{o} f_{A B}^{(2)} \Delta\right)^{2}} .
$$

Equation (20) is similar to the mass action equation obtained by SJ (Eq. (28) of Ref. 18), with the only difference being in the theory due to $\mathrm{SJ}$ the Mayer functions $f_{A B}^{(j)}$ are replaced by an exponential $f_{A B}^{(j)} \rightarrow e^{\varepsilon^{(j)} / k_{B} T}$. The advantage of Eq. (20) is that the exact non-associating limit $\rho=\rho_{o}$ is obtained, while in the approach of SJ this limit is obtained approximately.

A very interesting limit of Eq. (20) is for the case that the energy of the first bond in a cluster is zero $\varepsilon^{(1)} \rightarrow 0$. For this case we easily find two analytic solutions for the monomer density

$$
\begin{gathered}
\rho_{o} \rightarrow 1 / f_{A B}^{(2)} \Delta, \\
\rho_{o} \rightarrow \rho .
\end{gathered}
$$

For the density parameter $\sigma_{A}$ there is only a single limit

$$
\sigma_{A} \rightarrow \rho_{o} .
$$

Equation (23) shows that the density of spheres bonded once is vanishing in this limit, meaning any chains must be very long since there are few chain ends. Since it is unphysical for the monomer density to be greater than the total density $\rho_{o}>\rho$, Eq. (22) must be the correct solution for the case $\rho f_{A B}^{(2)} \Delta<1$. For the case $\rho f_{A B}^{(2)} \Delta>1$, Eq. (21) has the lowest free energy meaning it is the correct solution. At $\rho f_{A B}^{(2)} \Delta=1$ there is a transition point which we solve for as

$$
\left(\frac{\varepsilon^{(2)}}{k_{B} T}\right)_{t r}=\ln \left(\frac{\rho \Delta+1}{\rho \Delta}\right) .
$$

Below the transition point, $\varepsilon^{(2)} / k_{B} T<\left(\varepsilon^{(2)} / k_{B} T\right)_{t r}$, the fluid is composed of only monomers while after the transition the fluid is composed of a mixture of monomers and very long chains. At first it is surprising that the solution Eq. (21) would exist. However, it is easy to show the genesis of this solution. For the case of small $\varepsilon^{(1)}$ the function $\tilde{F}(1 \cdots s)$ will simplify to $\tilde{F}(1 \cdots s)=f_{A B}^{(1)}(12) f_{A B}^{(2)}(23) \cdots f_{A B}^{(2)}(s-1, s)$ where terms which contain products of $f_{A B}^{(1)}$ have vanished. At the point $\varepsilon^{(1)}=0$ these leading terms in $f_{A B}^{(1)}$ will also vanish with the exception of the case of an infinitely long chain whose contribution to $\Delta c^{(o)}$ is given by

$$
\frac{\Delta c_{\infty}}{V}=\frac{\sigma_{A} \sigma_{B}}{\rho_{o}} \frac{f_{A B}^{(1)}}{f_{A B}^{(2)}} \lim _{n \rightarrow \infty}\left(f_{A B}^{(2)} \rho_{o} \Delta\right)^{n} .
$$

The limit in Eq. (25) is evaluated as

$$
\lim _{n \rightarrow \infty}\left(f_{A B}^{(2)} \rho_{o} \Delta\right)^{n}=\left\{\begin{array}{lll}
\infty & \text { for } & f_{A B}^{(2)} \rho_{o} \Delta>1 \\
1 & \text { for } & f_{A B}^{(2)} \rho_{o} \Delta=1 \\
0 & \text { for } & f_{A B}^{(2)} \rho_{o} \Delta<1
\end{array},\right.
$$

which shows that for $f_{A B}^{(2)} \rho_{o} \Delta$ being infinitesimally larger than 1 , the limit diverges. This diverging limit tames the vanishing $f_{A B}^{(1)}$ resulting in a finite $\Delta c_{\infty}$ and the solution of the monomer density given by $\rho_{o}=1 / f_{A B}^{(2)} \Delta$. As will be shown in Sec. IV, the very simple relations given by Eqs. (21)-(24) are surprisingly accurate.

To make specific comparisons with simulations, now for $\varepsilon^{(1)}>0$, we will need monomer fractions $X_{o}=\rho_{o} / \rho$, fraction bonded once $X_{1}$

$$
X_{1}=\left(\rho_{A}+\rho_{B}\right) / \rho=2\left(\sigma_{A} / \rho-X_{o}\right)
$$

and the fraction bonded at both sites

$$
X_{2}=1-X_{o}-X_{1} .
$$

Finally the excess internal energy is obtained as

$$
\frac{E}{V}=\frac{\partial}{\partial \beta}\left(\frac{\beta A}{V}\right)=\rho \frac{\partial}{\partial \beta} \ln \rho_{o}-\frac{\partial}{\partial \beta} \sigma_{A},
$$

where $\beta$ is the inverse temperature $\beta=1 / k_{B} T$.

\section{SIMULATIONS}

To test the theory we perform new Monte Carlo simulations in the canonical ensemble for molecules which interact with the potential given by Eqs. (1)-(3). We choose potential parameters $\theta_{c}=27^{\circ}$ and $r_{c}=1.1 d$ such that each association site is singly bondable and place the sites on opposite poles of the sphere (see Fig. 1). The simulations are performed using standard methodology. ${ }^{25}$ The simulations were allowed to equilibrate for $N_{p} \times 10^{6}$ configurations and averages were taken over another $N_{p} \times 10^{6}$ configurations. A trial configuration was generated by displacing and rotating a sphere. For each simulation we used $N_{p}=864$ associating hard spheres. While in general having triplet contributions to the system energy can significantly increase computation time, for the current potential we simply needed to keep track of the number of spheres bonded twice, which added little computation time as compared to the pairwise additive system.

\section{RESULTS}

In this section we compare theoretical and simulation predictions. We begin our discussion with Fig. 3 where we compare theoretical and simulation predictions for the fraction of spheres bonded $k$ times $X_{k}$ and the excess internal energy $E^{*}=E / N_{p} k_{B} T$. We consider two general cases. In case I we set $\varepsilon^{(1)}=7 k_{B} T$ and vary $\varepsilon^{(2)}$ and for case II we fix 

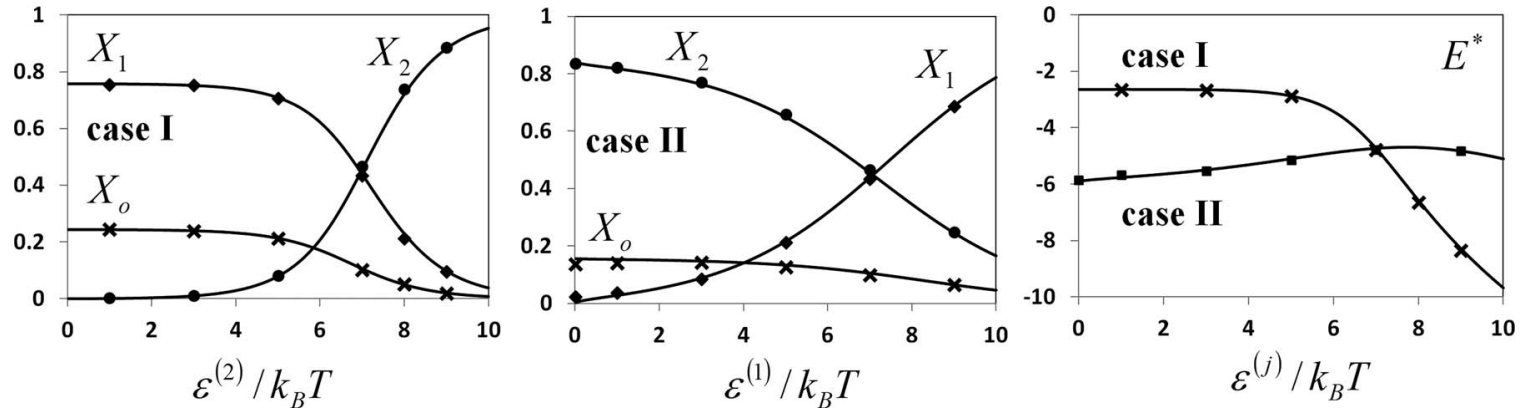

FIG. 3. Comparison of theoretical predictions (curves) and simulation results (symbols) for the fraction of molecules bonded $k$ times $X_{k}$ for case I - left panel and case II - center panel. The excess internal energy for both cases is given in the right panel. Each case is at a density of $\rho^{*}=0.6$. In case I $\varepsilon^{(1)}=7 k_{B} T$ and $\varepsilon^{(2)}$ is varied while in case II $\varepsilon^{(2)}=7 k_{B} T$ and $\varepsilon^{(1)}$ is varied. In the right panel $j=2$ for case I and $j=1$ for case II.

$\varepsilon^{(2)}=7 k_{B} T$ and vary $\varepsilon^{(1)}$. For each case we use a density of $\rho^{*}=\rho d^{3}=0.6$. We begin our discussion with case I. For $\varepsilon^{(2)}=0$, there is no energetic benefit for a sphere to bond twice which results in $X_{2} \rightarrow 0$. Increasing $\varepsilon^{(2)}$ we see a steady increase in $X_{2}$ and the fractions $X_{1}$ and $X_{o}$ remain nearly constant until $\varepsilon^{(2)} \sim 5 k_{B} T$ at which point they decline sharply. The excess internal energy also remains approximately constant until $\varepsilon^{(2)} \sim 5 k_{B} T$ and then begins to decrease. Theory and simulation are in near perfect agreement.

Now considering case II, we set the energy $\varepsilon^{(2)}$ and vary $\varepsilon^{(1)}$. We note the opposite behavior for the fraction $X_{2}$ as compared to case I. Increasing $\varepsilon^{(1)}$ decreases $X_{2}$ while increasing $X_{1}$. This behavior results from the fact that for small $\varepsilon^{(1)}$ the absolute value of the energy per bond is much lower for the first bond in an associated cluster than all remaining bonds in that cluster. For this reason the system minimizes $X_{1}$. The behavior of $E^{*}$ is remarkable for this case. The internal energy increases with increasing $\varepsilon^{(1)}$ until $\varepsilon^{(1)} \sim 7.8 k_{B} T$ at which point there is a maximum and $E^{*}$ begins to decrease. It is counter intuitive that increasing $\varepsilon^{(1)}$ could result in an increase in energy. Again, theory and simulation are in excellent agreement. We also performed calculations for the monomer fraction $X_{o}$ and excess internal energy $E^{*}$ using the theory of SJ; ${ }^{18}$ these predictions coincided nearly exactly to the calculations performed with the approach presented in this work. No prescription is given by SJ for the calculation of the fractions $X_{1}$ and $X_{2}$.

Now we focus on the specific case where $\varepsilon^{(1)}=0$. For this case the monomer fraction is given by Eqs. (21) and (22). We compare predictions of these simple equations to simulation results for the monomer fractions in Fig. 4 at densities $\rho^{*}=0.2$ and 0.6. The locations where the solid curves cross the long dashed curve are the transition points predicted by Eq. (24). Before the transition the monomer density is given by Eq. (22), and after the transition by Eq. (21). We also include theoretical predictions from the approach of SJ. ${ }^{18}$ As can be seen, both simulation and SJ predict a smooth transition while the transition predicted by the current approach is sharp. This discrepancy is at the heart of the difference between our approach and the approach of SJ. In Wertheim's theory the exact non-associating limit $\rho_{o}=\rho$ is obtained. That is, if the association energy is zero there is no associa- tion. The exception to this rule, is for the case presented in this paper for bond cooperativity where $\varepsilon^{(1)}=0$ and the monomer density is given by Eq. (21). The theory of SJ does not obey this non-associating limit. In their approach associated chains of spheres can exist even when all association energies are zero due to the fact that association has a purely geometric definition, meaning the exact reference equation of state is not recovered. Molecular simulation also uses this strictly geometric definition of bonding. For these reasons, both the theory of SJ and molecular simulation show a smooth transition while the current approach shows a sharp transition.

Another prediction of the current approach was that after the transition the fluid would be composed of a mixture of monomers and very long chains. The inset of Fig. 4 shows simulation results for the average chain length of associated chains (not including monomers) $L$ as a function of $\varepsilon^{(2)} / k_{B} T$ and a density $\rho^{*}=0.2$. As can be seen, after the transition $L$ grows rapidly as $\varepsilon^{(2)} / k_{B} T$ is increased. At an energy $\varepsilon^{(2)}=9 k_{B} T$ the monomer fraction is found to be $X_{o} \approx 0.1$ with the fraction bonded once as $X_{1} \approx 0.016$ giving an average chain length of associated clusters as $L \approx 117$. This shows that the fluid is primarily composed of monomers and long chains as predicted by the theory.

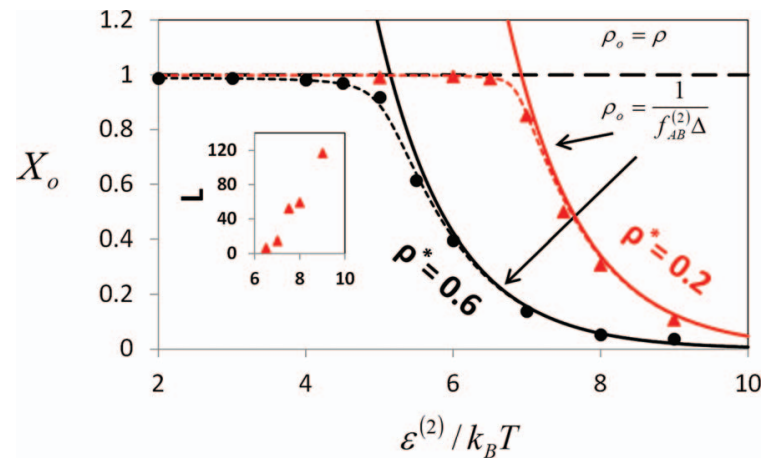

FIG. 4. Monomer fractions versus $\varepsilon^{(2)} / k_{B} T$ for the case $\varepsilon^{(1)}=0$. Symbols give simulation results (triangles $-\rho^{*}=0.2$ and circles $-\rho^{*}=0.6$ ). Long dashed curve gives the theoretical solutions $\rho=\rho_{o}$ and solid curves give the solution $\rho_{o}=1 / f_{A B}^{(2)} \Delta$. Short dashed curves give theoretical results from the theory of SJ. ${ }^{18}$ The inset gives the simulated values of the average chain length of associated clusters (excluding monomers) versus $\varepsilon^{(2)} / k_{B} T$ at $\rho^{*}=0.2$. 


\section{DISCUSSION AND CONCLUSIONS}

We have extended Wertheim's theory to account for bond cooperativity in two site associating fluids by the development of a new perturbation theory. This was accomplished in an intuitive manner, guided by the structure of Wertheim's TPTN. The new theory was tested against Monte Carlo simulation results and was found to be accurate. For the case that the first bond of an associated cluster had zero energy $\varepsilon^{(1)}=0$, a simplified form of the theory was obtained predicting that a sharp transition existed between a fluid composed of only monomers and a fluid composed of monomers and very long chains. This transition was also observed by molecular simulation; however, the simulated transition was smooth. The discrepancy between theory and simulation is attributable to the different definitions of association.

The theory of $\mathrm{SJ}^{18}$ was also found to be accurate. This was the first time this approach had been compared to molecular simulation. An advantage of the approach developed here is that the exact non-associating limit is obtained, while in the approach of SJ this approach is obtained approximately. That said, the two theories given nearly identical predictions when all association energies are finite.

The incorporation of bond cooperativity into Wertheim's multi-density formalism allows for the development of a variety of equations of state for fluids which exhibit bond cooperativity. For instance, extension of the current approach to include the effects of ring formation is straight forward. Rings were first considered in the context of Wertheim's theory by SJ. ${ }^{26}$ More recently Marshall and Chapman ${ }^{22,27}$ developed an approach which accounts for the effect of bond angle on ring formation. Since each sphere in a ring is bonded twice, all bonds will be given Mayer functions $f_{A B}^{(2)}(12)$. For this case $\Delta c^{(o)}$ will be composed of contributions for chain and ring formation as

$$
\frac{\Delta c^{(o)}}{V}=\frac{\sigma_{A} \sigma_{B} f_{A B}^{(1)} \Delta}{1-\left(f_{A B}^{(2)}-f_{A B}^{(1)}\right) \rho_{o} \Delta}+\sum_{n=3}^{\infty} \frac{\left(f_{A B}^{(2)} \rho_{o} \Delta\right)^{n}}{n d^{3}} I_{r}^{(n)} .
$$

The second term in Eq. (30) gives the sum over all ring sizes and $I_{r}^{(n)}$ is the ring integral which depends on bond angle and is given in Ref. 22. For the potential parameters used in this paper ${ }^{28}$ and the association sites in an axisymmetric location, ${ }^{22}$ ring formation is negligible. However, if one were to decrease the bond angle between the association sites, or increase the size and range of the association sites, ring formation would become significant. Other extensions include the effects of cooperativity on double bonding between molecules, ${ }^{22,26}$ for the case that a single site can bond twice ${ }^{29,30}$ and for mixtures of molecules with spherically symmetric and anisotropic interactions. ${ }^{31}$ Taking the logic used in this paper further, we can consider the case where the $k$ th bond in a chain receives an energy $-\varepsilon^{(k)}$ and is assigned a Mayer function $f_{A B}^{(k)}$. For this case the functions $\tilde{f}(1 \cdots s)$ would be given by

$$
\tilde{f}(1 \cdots s)=\prod_{k=1}^{s-1} f_{A B}^{(k)} \mathrm{O}(k, k+1) .
$$

This would be a more realistic model for $\mathrm{HF}^{12}$ than the model considered in this paper, due to the fact that the bonding energy changes as a function of chain length beyond what is given by Eqs. (1) and (2). Combining Eqs. (13) and (31) gives a generalized theory for bond cooperativity in chain formation for two site associating fluids.

Lastly, Kalyuzhnyi and Stell ${ }^{32}$ developed a cluster expansion similar to Wertheim's except for the densities are defined in a way which is convenient for molecules which interact with spherically symmetric association interactions (e.g., ions). This approach has also been applied in the development of an equation of state for dipolar hard spheres ${ }^{33}$ as well as patchy colloids with patches which bond twice. ${ }^{34}$ It should be possible to include bond cooperativity in these theories also.

\section{ACKNOWLEDGMENTS}

The financial support of The Robert A. Welch Foundation Grant No. C-1241 is gratefully acknowledged.

${ }^{1} \mathrm{G}$. A. Jeffrey, An Introduction to Hydrogen Bonding (Oxford University Press, New York, 1997).

${ }^{2}$ K. A. Dill, Biochemistry 29(31), 7133-7155 (1990).

${ }^{3}$ P. Cordier, F. Tournilhac, C. Soulié-Ziakovic, and L. Leibler, Nature (London) 451(7181), 977-980 (2008).

${ }^{4}$ M. Wertheim, J. Stat. Phys. 35(1), 19-34 (1984).

${ }^{5}$ M. Wertheim, J. Stat. Phys. 35(1), 35-47 (1984).

${ }^{6}$ M. Wertheim, J. Stat. Phys. 42(3), 459-476 (1986).

${ }^{7}$ M. Wertheim, J. Stat. Phys. 42(3), 477-492 (1986).

${ }^{8}$ M. Wertheim, J. Chem. Phys. 87, 7323 (1987).

${ }^{9}$ W. G. Chapman, G. Jackson, and K. E. Gubbins, Mol. Phys. 65(5), 10571079 (1988).

${ }^{10}$ E. A. Müller and K. E. Gubbins, Ind. Eng. Chem. Res. 40(10), 2193-2211 (2001).

${ }^{11}$ Z. Feng, A. Bymaster, C. Emborsky, D. Ballal, B. Marshall, K. Gong, A. Garcia, K. R. Cox, and W. G. Chapman, J. Stat. Phys. 145(1), 467-480 (2011).

${ }^{12}$ L. Rincón, R. Almeida, D. García-Aldea, and H. Diez y Riega, J. Chem. Phys. 114, 5552 (2001).

${ }^{13}$ S. J. Barlow, G. V. Bondarenko, Y. E. Gorbaty, T. Yamaguchi, and M. Poliakoff, J. Phys. Chem. A 106(43), 10452-10460 (2002).

${ }^{14}$ H. Guo and M. Karplus, J. Phys. Chem. 98(29), 7104-7105 (1994).

${ }^{15}$ K. Stokely, M. G. Mazza, H. E. Stanley, and G. Franzese, Proc. Natl. Acad. Sci. U.S.A. 107(4), 1301-1306 (2010).

${ }^{16}$ P. Muthukumaran, R. L. Brinkley, and R. B. Gupta, AIChE J. 48(2), 386392 (2002).

${ }^{17}$ B. A. Veytsman, J. Phys. Chem. 97(28), 7144-7146 (1993).

${ }^{18}$ R. P. Sear and G. Jackson, J. Chem. Phys. 105(3), 1113 (1996).

${ }^{19}$ W. Bol, Mol. Phys. 45, 605 (1982).

${ }^{20}$ G. Jackson, W. G. Chapman, and K. E. Gubbins, Mol. Phys. 65(1), 1-31 (1988).

${ }^{21}$ N. Kern and D. Frenkel, J. Chem. Phys. 118, 9882 (2003).

${ }^{22}$ B. D. Marshall and W. G. Chapman, Phys. Rev. E 87, 052307 (2013).

${ }^{23}$ W. G. Chapman, Ph.D. thesis, Cornell University, Ithaca, NY, 1988.

${ }^{24}$ J. P. Hansen and I. R. McDonald, Theory of Simple Liquids (Academic press, 2006).

${ }^{25}$ D. Frenkel and B. Smit, Understanding Molecular Simulation: From Algorithms to Applications (Academic press, 2001).

${ }^{26}$ R. P. Sear and G. Jackson, Mol. Phys. 82(5), 1033-1048 (1994).

${ }^{27}$ B. D. Marshall and W. G. Chapman, J. Chem. Phys. 139, 054902 (2013).

${ }^{28}$ J. M. Tavares, L. Rovigatti, and F. Sciortino, J. Chem. Phys. 137(4), 044901 (2012).

${ }^{29}$ B. D. Marshall, D. Ballal, and W. G. Chapman, J. Chem. Phys. 137(10), 104909 (2012)

${ }^{30}$ B. D. Marshall and W. G. Chapman, J. Chem. Phys. 138, 044901 (2013).

${ }^{31}$ B. D. Marshall and W. G. Chapman, J. Chem. Phys. 139, 104904 (2013).

${ }^{32}$ Y. V. Kalyuzhnyi and G. Stell, Mol. Phys. 78(5), 1247-1258 (1993).

${ }^{33}$ Y. Kalyuzhnyi, I. Protsykevytch, and P. Cummings, EPL 80, 56002 (2007).

${ }^{34}$ Y. V. Kalyuzhnyi, B. D. Marshall, W. G. Chapman, and P. T. Cummings, J. Chem. Phys. 139, 044909 (2013). 